\title{
A Study of Vending Machine Interface System and User Behavior on Multi-Interface Vending Machine Improved Design
}

\author{
Ping $\mathrm{Li}^{* 1, \mathrm{a}}$, \\ ${ }^{1}$ Beijing Institute of Technology, 5 South Zhongguancun \\ Street, Haidian District, Beijing, China \\ ali_ping8888@hotmail.com
}

\begin{abstract}
Vending machines must offer efficient service with zero tolerance for human error, require no user training and be accessible to a wide range of users. User behavior analysis by product interface guides the user's behavior is the key to solving some practical interaction design problems. This study based on Multimedia technology, evaluates the user experience of the multi-interface vending machines in light of recent research results in human computer interaction. Several design problems are identified and improvements are suggested.
\end{abstract}

Keywords- user behavior interaction design touch sensitive display merchandise display, multi-interface human computer interaction

\section{INTRODUCTION}

Vending machines have been around for decades, has grown sharply during the recent decade, due to low-cost hardware and high cost of human staff [1]. A well-designed Vending machines can help increase the profits of an organization and contribute to customer satisfaction,it eliminates all unnecessary salesman with strange exchange between the owner. Since the vending machines will become interpersonal communication exchange between man and machine, and then how to make the communication more quickly and reliably process? The design of user interfaces for public kiosks is challenging. Vending machine or kiosk user interface must be self explanatory and accessible without prior training. Furthermore, few assumptions can be made about the skill level and knowledge of the users. [2] Users may suffer from cognitive interference due to stress and the user interface must support the user task and minimize the probability of error.

\section{THE PROCESS OF USER BEHAVIOR}

\section{A. Select Product - Quote}

1) Code Input to Select Goods. Many vending machines are in accordance with rows and columns to organize merchandise. If you want to select an item, then you need to input the code of the goods where the row and column of figures. The basic problem is the left half of the vending machine with a large display window for the display of goods, the operating system on the right side, which leads from the right side of the operating system the user is

\author{
Cheng $\mathrm{Li}^{2, \mathrm{~b}}$ \\ ${ }^{2}$ Tianjin University 92 Weijin Road, Nankai District, \\ Tianjin, China \\ bli_zheng_yirong@sina.com
}

moved to the left to select goods, then move to enter the code on the right side. Vending machines are not home consumer electronics, build human and machine in prolonged contact and use of muscle memory, even in the possibility of using the initial stages of a malfunction, but with the gradual adaptation of the machine, the user can reach the so-called the "eyes closed operations" realm; and vending machines is public facilities, the use of user density is insufficient to form muscle memory, so each time you use this move in repeating the process, so long distance visual movement is likely to cause short-term memory errors, causing malfunction, you get a goods that you does not want to .

2) Intuitive Buttons to Select Goods. This design is relatively straightforward, each of goods has a button with the following commodity prices, just press the button on the bottom of the commodity can be selected merchandise. A good solution to short-term memory losing, customer could get the right goods. The only shortcoming is too much bottoms resulting in the overall structure of the machine bloated.

3) The touch sensitive display to Select Goods. All prices and other commodities as well as focus on the touch screen display, the touch-screen prompts to select merchandise, at a glance, to prevent misuse.

Japan recently introduced a smart vending machine, it shows different goods, sensors installed on vending machines will automatically determine the age and gender of customers, and for customers to display the corresponding recommended product depending on the season and time of the change, to the young Women recommended the addition of vitamins drinks or tea, the men recommended nutritional drink or coffee. When nobody in front of the vending machine, it will become a giant cartoon characters, sends information or promote the new product. [3]. The touch sensitive display has emerged as a practical input technology compared to physical keyboards, speech recognition[4]. 


\subsection{Payment- Change}

Existing vending machines payment are cash (banknotes, coins),credit card (debit card).Touch-screen interface can be extended payment.

1) Mobile Payment. By input phone number and verification code to complete the payment of the phone balance.

2) Online Payment. Payment completed by connecting online banking.

3) QR Code Bill. Each item in vending machines has a QR code, the formation of an electronic bill by scanning the QR code, you can set up online banking with late payments or unity at any convenient time to pay the bill.

\subsection{Pick Up the Goods}

The traditional way of vending machines to drop down the products directly, which may lead to some product packaging damaged when dropped, carbonated beverages could be splash after the fall of the impact when it opened. Pick up mouth at the bottom of vending machines, as the machine will you buy goods rudely thrown at your feet, consumers should leaned down to pick up the merchandise, it is a failure of interface experience.

Pickup mouth redesigned will be located in the hand touch region for adults standing position to avoid bend over.

\subsection{The Following Case Study of A Specific Vending Machine Interface Design}

This study focuses on a multi interface Vending Machine or kiosk type, namely Asian food kiosk .Typically such machines are equipped with a touch sensitive color display [5] which is used for most of the interactions. The touch sensitive display has emerged as a practical input technology compared to physical keyboards, speech recognition[6], interaction through personal mobile devices [7] or interaction through kiosk sensors, can be used to build tangible user interfaces.

\subsection{Change a Single User Interface to Multi-Interface}

The traditional vending machine is a single interface, only one operation, This design case Change the traditional cube form into a cylinder, so that single interface turn to a omni directional interface, up to five people operation.
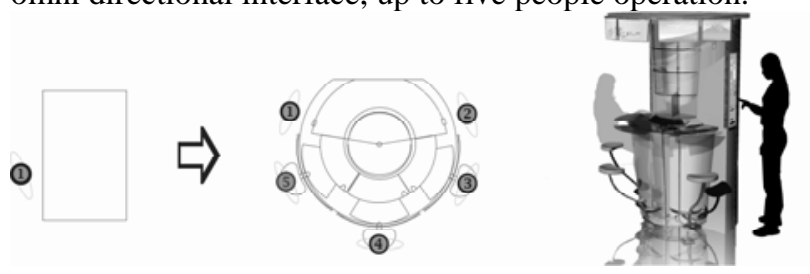

Figure 1. Change a single user interface to multi-interface

\subsection{Choose a variety of ways to buy. Take out or eat here}

To provide consumers with a variety of options that can be packed away, or you can sit down and enjoy a meal. You can sit down like real sushi shop in front of the rotation by the same electronic screen to select their favorite delicacy, lots of fun.

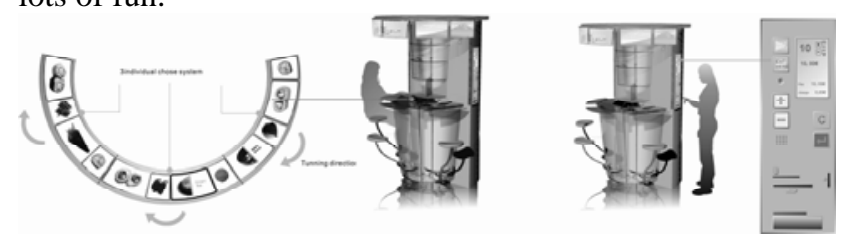

Figure 2. Take out or eat here.

\subsection{Asian Food Kiosk Interface Design, Take Out Mood.}

Consumers in front of the touch sensitive display to select takeaway mode, then select drinks, sushi and dim sum one by one. Payment can be in many ways, the figure payment is to scan two-dimensional code, two-dimensional code is stored to the phone bill, and when it is convenient payments. Vending machines will be automatically packaged goods, consumers can easily get the goods shipped from the pickup mouth below touch sensitive display. display fonts are unified page is not more than two kinds of fonts, background colors with low saturation, in the foreground color saturation, focus on tips for a small area of high-saturation color, structured, reduce visual errors. Pickup mouth redesigned will be located in the hand touch region.

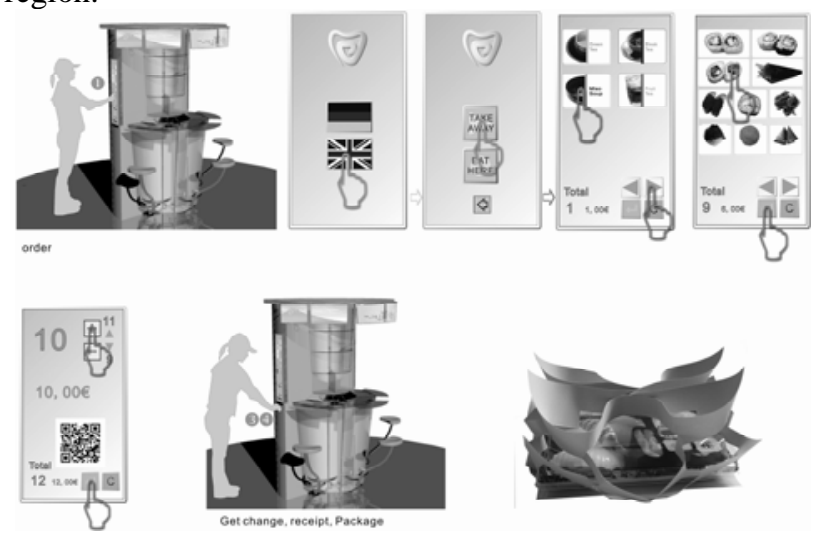

Figure 3. Take out Mood

\subsection{Asian Food Kiosk Interface Design, Eat Here mood.}

Consumers choose "Eat Here" in the electronic touch-screen , pay the appropriate amount of a single product, dine sitting area, implant rotary sushi sales concept, increase the user experience, through a circular rotating touch screen ordering drink and food. Machine will deliver you the goods by the window in front of table, you can easily enjoy your meals. In front of the machine has three seats, providing up to three people at a time. With one or two friend to enjoy a very pleasant meal, you can open the table after dinner push the plate in to recycle, Reflects concern for the environment. 

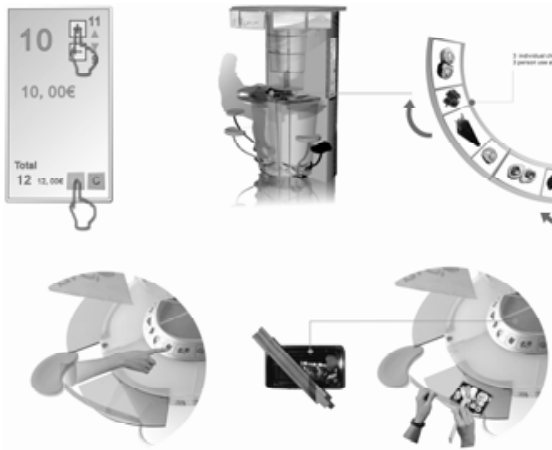

Figure 4. Eat here Mood

\section{SUMMARY}

The analysis revealed several weaknesses in the existing user interface and a number of improvements based on the proposed design heuristics were suggested.

The improvements of Asian Food Kiosk user interface design highest priority include:

Change a single user interface to multi-interface,

Variety of ways to buy,

Avoid unnecessary visual elements,

Dine sitting area, implant rotary sushi sales concept, increase the user experience.
When designing such systems it is important to apply design processes where the possible design space is adequately explored by the design team such that one can maximize the accessibility of the product accompanied by user-centric evaluations.

\section{REFERENCES}

[1] L. L. Tung and J. H. Tan, "A model for the classification of information kiosks in Singapore," International Journal of Information Management, Vol. 18, 1998, pp.255-264.

[2] Frode Eiea Sandnes, "User Interface Design for Public Kiosks: An Evaluation of the Taiwan High Speed Rail Ticket Vending Machine" Journal of International Science and Engineering 26, (2010)307-321

[3] Information on

http://japan-magazine.jnto.go.jp/zh/1208_vending.html

4] C. Guinn and R. Hubal, "An evaluation of virtual human technology in informational kiosks," in Proceedings of the 6th International Conference on Multimodal Interfaces, 2004, pp. 297-302.

[5] P. A. Albinsson and S. S. Zhai, "High precision toch screen interaction," in Proceedings of the SIGCHI Conference on Human Factors in Computing Systems, 2003,pp. 105-112.

[6] L. Lamel, S. Bennacef, J. L. Gauvain, H. Dartigues, and J. N. Temem, "User evaluation of the MASK kiosk," Speech Communication, Vol. 38, 2002, pp. 131-139.

[7] Huang, K. Pulli, and L. Rudolph, "Kimono: kiosk-mobile phone knowledge sharing system," in Proceedings of the 4th International Conference on Mobile and Ubiquitous Multimedia, 2005, pp. 142-149. 